\title{
INVENTORY AND CONSERVATION OF BREEDING WATERBIRDS AT OJO DE LIEBRE AND GUERRERO NEGRO LAGOONS, BAJA CALIFORNIA SUR, MEXICO
}

\section{INVENTARIO Y CONSERVACIÓN DE LA AVIFAUNA ACUÁTICA REPRODUCTORA DE LAS LAGUNAS OJO DE LIEBRE Y GUERRERO NEGRO, BAJA CALIFORNIA SUR, MÉXICO}

\author{
Aradit Castellanos* \\ Federico Salinas \\ Alfredo Ortega-Rubio \\ Centro de Investigaciones Biológicas del Noroeste, S.C. \\ Apartado postal 128 \\ La Paz, C.P. 23000, Baja California Sur, México \\ * E-mail: arcas@cibnor.mx
}

Recibido en junio de 2000; aceptado en abril de 2001

\begin{abstract}
Surveys made in 1997/98 at the lagoons of Ojo de Liebre (Scammon's Lagoon) and Guerrero Negro, Baja California Sur, Mexico, showed that changes have occurred since 1926 in the species composition of the community of breeding waterbirds, in the arrangement of their nesting substrate and in their population size. Of 17 species breeding in the area in 1926, we found 12 nesting there during our survey. Additional data from other researchers allowed us to conclude that 16 species have nested in the area after 1984. Only one species (Sterna elegans) has not been reported nesting; however, it is still considered to be a local breeder. Three species (Egretta alba, Larus atricilla and Falco peregrinus) became new established breeders. Most of the species that breed on the islands within Scammon's Lagoon have a stable population or have increased in number. None of the egret species breeding in 1926 in marshes outside the islands currently nest there. Four species (Sterna antillarum, Charadrius wilsonia, Charadrius alexandrinus and Rallus longirostris) breed on the islands or at other sites in the lagoons and vicinity. Despite development in the region, the natural wetlands and open-water coverage remain almost unchanged. Natural nesting substrates are mostly confined to the islands and are vulnerable to land predators, wave erosion and direct human disturbance. These factors may have caused the shifting of nesting spots from one island to another and to other suitable nesting sites on the lagoons, the urban landscape, the saltworks and man-made structures. Breeding waterbirds have been granted limited protection through reserves and single-species conservation programs. We suggest guidelines to enhance their protection as a community in the context of El Vizcaíno Biosphere Reserve.
\end{abstract}

Key words: Ojo de Liebre, Baja California Sur, breeding waterbirds. 
Ciencias Marinas, Vol. 27, No. 3, 2001

\section{RESUMEN}

Inventarios realizados entre 1997 y 1998 en las lagunas Ojo de Liebre y Guerrero Negro, Baja California Sur, México, mostraron que desde 1926 han ocurrido cambios en la composición de especies de aves acuáticas reproductoras, en la distribución de sus substratos de anidamiento y en el tamaño de sus poblaciones. De 17 especies que anidaban en el área en 1926, encontramos 12 anidando ahí durante nuestro inventario. Datos adicionales de otros investigadores nos permiten concluir que 16 especies han anidado en al área después de 1984. Sólo una de las especies históricamente reportadas (Sterna elegans) no ha sido vista anidando; sin embargo, no se descarta como un reproductor local. Tres especies (Egretta alba, Larus atricilla y Falco peregrinus) se establecieron como nuevos reproductores. La mayoría de las especies que anidan en las islas dentro de la Laguna Ojo de Liebre tienen poblaciones estables o incrementaron sus números. Ninguna de las especies de garzas que en 1926 anidaban en las marismas fuera de las islas, se encontró anidando ahí en nuestro inventario. Cuatro especies (Sterna antillarum, Charadrius wilsonia, Charadrius alexandrinus y Rallus longirostris) anidan fuera de las islas, en otros sitios de las lagunas. No obstante el desarrollo de la región, la cobertura de los humedales naturales y de aguas abiertas permanece casi sin cambios. Los substratos de anidamiento naturales están confinados, en su mayor parte, a las islas y son vulnerables a los depredadores terrestres, a la erosión por el oleaje y a la perturbación humana directa. Estos factores pudieron haber causado el cambio de zonas de anidamiento de una isla a otra y hacia algunos sitios de anidamiento adecuados en las lagunas, al paisaje urbano, la salina y en estructuras antropogénicas. Las aves acuáticas reproductoras obtuvieron protección incipiente a través del establecimiento de reservas y de programas de conservación de especies singulares. Sugerimos algunos lineamientos para fortalecer su protección como comunidad en el contexto de la Reserva de la Biósfera El Vizcaíno.

Palabras clave: Ojo de Liebre, Baja California Sur, aves acuáticas.

\section{INTRODUCTION}

Scammon's (Ojo de Liebre) and Guerrero Negro lagoons comprise one of the few relatively well-preserved wetlands on the west coast of Mexico (Wilson and Ryan, 1996). Because of the variety of habitats, species richness and size of the populations of migratory and resident waterbirds found in this area, the Mexican government recognized it as one of priority for conservation, establishing a wildlife refuge in 1972 and including both lagoons in the El Vizcaíno Biosphere Reserve in 1988 (Breceda et al., 1995).

These lagoons are an important breeding location for many waterbird species on the west coast of the Baja California peninsula (see Bancroft, 1927; Grinnell, 1928). Despite the

\section{INTRODUCCIÓN}

Las lagunas Ojo de Liebre y Guerrero Negro son de los pocos humedales relativamente bien conservados en la costa occidental de México (Wilson y Ryan, 1996). Debido a la variedad de hábitats, a su riqueza de especies y a la abundancia de sus poblaciones de aves acuáticas migratorias y residentes, el gobierno mexicano las ha reconocido como prioritarias para la conservación, estableciendo un refugio de vida silvestre en 1972 e incluyendo ambas lagunas en la Reserva de la Biósfera El Vizcaíno en 1988 (Breceda et al., 1995).

Estas lagunas integran una zona importante de reproducción para muchas especies de aves acuáticas en la costa occidental de la península de Baja California (Bancroft, 1927; Grinnell, 
conservation value of this area, its avifauna is poorly known. Bancroft (1927) provided the first inventory of breeding waterbird species at Scammon's Lagoon and some general references about their nesting habitat arrangement. Since then, most of the ornithological information produced for the area has focused on aspects of the reproductive biology and ecology of a scant number of species of concern, such as the osprey (Kenyon, 1947; Jehl, 1977; Henny and Anderson, 1979; Castellanos and Ortega-Rubio, 1995), the peregrine falcon (Castellanos et al., 1994a, 1997) and the laughing gull (Castellanos et al., 1994b). However, other authors provide a wider perspective of the waterbirds in the region. Everett and Anderson (1991) made a review of the status of some species at Scammon's Lagoon; Massey and Palacios (1994) provide information on the abundance of breeding waterbirds, the use of their nesting sites and the main habitat threats. Recently, Carmona and Danemann (1998), and Danemann and Carmona (2000) documented spatiotemporal distribution patterns of the waterbirds at the Guerrero Negro saltworks, an area not included in our study. Despite these studies, there is a lack of current and detailed information on the breeding waterbirds in the lagoons, because after the pioneering work of Bancroft (1927), no other focused, comprehensive survey has been made.

The determination and description of past and current patterns of occurrence, distribution and abundance of this community contributes to enhance the knowledge of its ecology and the natural history of the area, to visualize the role of man in its changes, and provides a territorial base of information to support conservation measures. The last is important because conservation measures, to be effective, should be geographically oriented (see Soulé, 1991).

In this study we synthetize historical information on the breeding waterbirds of
1928). A pesar de su valor de conservación, su avifauna es pobremente conocida. Bancroft (1927) proporcionó el primer inventario de las especies de aves acuáticas reproductoras en la Laguna Ojo de Liebre y referencias generales de la distribución de sus hábitats de anidamiento. La mayor parte de la información ornitológica sobre el área generada desde entonces, está enfocada a aspectos de la biología y ecología reproductiva de un escaso número de especies de interés, como el águila pescadora (Kenyon, 1947; Jehl, 1977; Henny y Anderson, 1979; Castellanos y Ortega-Rubio, 1995), el halcón peregrino (Castellanos et al., 1994a, 1997) y la gaviota reidora (Castellanos et al., 1994b). Sin embargo, otros autores proveen una perspectiva más amplia de la avifauna acuática de la región. Everett y Anderson (1991) hicieron una revisión sobre el estatus de algunas especies de la Laguna Ojo de Liebre; Massey y Palacios (1994) proporcionaron información sobre la abundancia de las aves acuáticas reproductoras, del uso de sitios de anidamiento y de las amenazas principales a su hábitat. Recientemente, Carmona y Danemann (1998) y Danemann y Carmona (2000) documentaron los patrones espaciotemporales de distribución de la avifauna acuática dentro de la salina de Guerrero Negro, un área no incluida en nuestro estudio. No obstante estas investigaciones, se carece de información actualizada y detallada sobre la avifauna acuática reproductora en las lagunas, ya que desde el trabajo pionero de Bancroft (1927) hasta la fecha, no se ha hecho otro inventario completo y enfocado de la misma.

El determinar y describir los patrones pasados y actuales de ocurrencia, distribución y abundancia de esta comunidad contribuye a ampliar el conocimiento de su ecología y de la historia natural del área, a visualizar el papel del hombre en sus cambios, y provee una base territorial de información para sustentar medidas de conservación. Este último 
Scammon's and Guerrero Negro lagoons and vicinity. We report results of ground surveys made during 1997/98 in the area. We provide data on the species present, colony distribution and breeding pair numbers, and compare our results with those of Bancroft (1927), Massey and Palacios (1994) and with unpublished government reports of surveys and inventories from the late 1970s and early 1980s. We discuss the changes of the distribution and use of local nesting sites and the factors contributing to this. Also, we examine the main problems confronted by this group of species, such as habitat change, depredation and direct human perturbation, as well as approaches to its protection implemented in the region. We suggest guidelines for conservation of this community in the context of El Vizcaíno Biosphere Reserve.

\section{DESCRIPTION OF THE AREA AND METHODS}

Scammon's and Guerrero Negro lagoons are located on the west coast of the Baja California peninsula, Mexico (fig. 1). Both lagoons together cover about $500 \mathrm{~km}^{2}$. The main type of vegetation around the lagoons is halophytic shrub. Shorelines are in part salt marshes, sand dunes, halophytic shrub and man-made brine pans. Inside Scammon's Lagoon, there are five small $\left(0.05\right.$ to $\left.7.5 \mathrm{~km}^{2}\right)$ flat islands, with sparse vegetation and fringes of marsh. Along the lagoon's natural wetlands and open waters there are about 37,500 ha of man-made brine pans of a saltworks, Exportadora de Sal, S.A. de C.V. (ESSA) (fig. 1). Additional descriptions of the lagoons can be found in Lewis and Ebeling (1974), and Saunders and Saunders (1981).

Between late December 1997 and June 1998, we made seven surveys by foot, boat or car at both lagoons and in the vicinity to locate waterbird nesting sites (including man-made aspecto es importante, ya que las medidas de conservación para ser efectivas deben estar geográficamente orientadas (Soulé, 1991).

En este estudio sintetizamos información histórica sobre las aves acuáticas reproductoras de las lagunas Ojo de Liebre y Guerrero Negro, y presentamos nuestros resultados de inventarios terrestres realizados entre 1997 y 1998. Proporcionamos información sobre las especies presentes, la distribución actual de las colonias y el número de parejas reproductoras, y comparamos nuestros resultados con los de Bancroft (1927), Massey y Palacios (1994) y con reportes gubernamentales no publicados de exploraciones e inventarios de finales de los años setenta y principios de los ochenta. Discutimos los cambios en la distribución y uso de los sitios de anidamiento y los factores que contribuyen a esto. Asimismo, examinamos los problemas principales que enfrentan este grupo de especies, tales como cambios en el hábitat, depredación y perturbación humana directa, así como los programas y acciones para su protección instrumentados en la región. Finalmente, sugerimos lineamientos para la conservación de esta comunidad en el contexto de la Reserva de la Biósfera El Vizcaíno.

\section{DESCRIPCIÓN DEL ÁREA Y MÉTODOS}

Las lagunas Ojo de Liebre y Guerrero Negro se localizan en la costa occidental de la península de Baja California, México (fig. 1). Ambas lagunas abarcan aproximadamente $500 \mathrm{~km}^{2}$. El principal tipo de vegetación alrededor de las lagunas es el matorral halófilo. Las costas están en parte constituidas por marismas salinas, dunas, matorral halófilo y bordos de estanques de producción de sal. Dentro de la Laguna Ojo de Liebre se encuentran cinco pequeñas islas $\left(0.05\right.$ a $\left.7.5 \mathrm{~km}^{2}\right)$ planas, con escasa vegetación y franjas de marismas. A lo largo de las marismas y aguas abiertas de las lagunas hay aproximadamente 37,500 ha de 

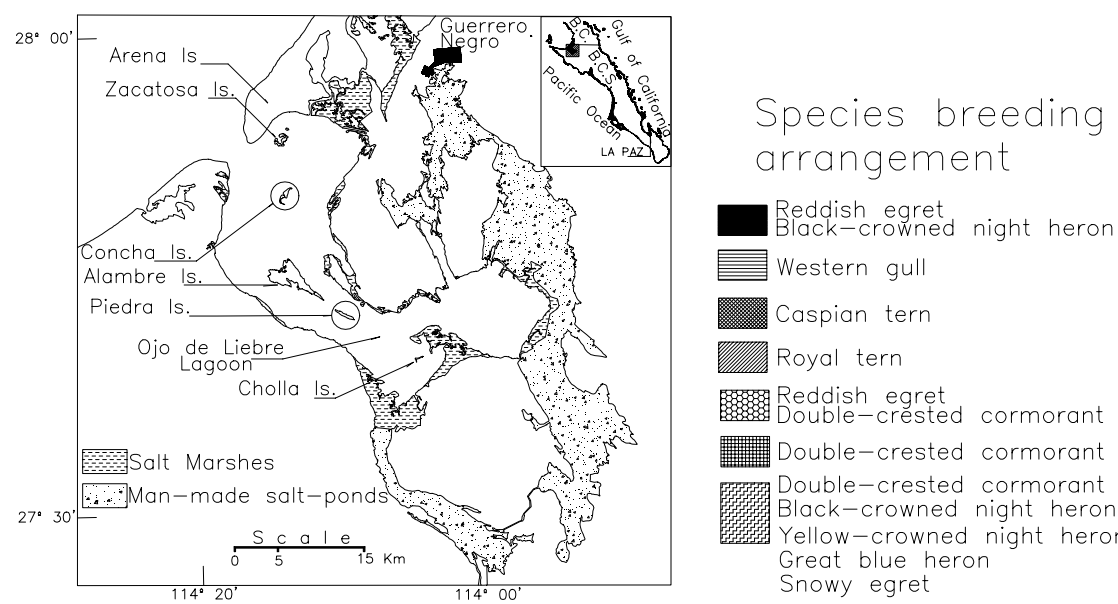

1) Caspian tern

WIIISR Royal tern

Frgatish egret

Double-crested cormorant

WFible-crested cormorant

Wlack-crowned night heron Great blue heron Snowy egret

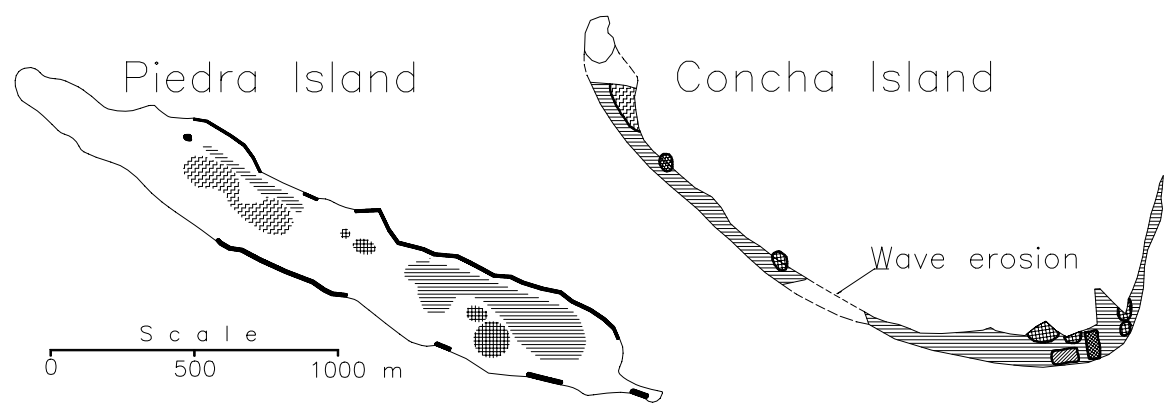

Figure 1. Distribution of the main reproductive colonies of waterbirds at Ojo de Liebre Lagoon, Baja California Sur, Mexico.

Figura 1. Distribución de las principales colonias reproductoras de aves acuáticas en la Laguna Ojo de Liebre, Baja California Sur, México

structures located inland suitable as nesting sites for raptors). Each survey spanned four days and was made by three observers. We recorded data on the species present and nesting sites, and nest content when possible. We mapped $(1: 50,000)$ nesting sites and estimated the number of breeding pairs.

Population size of non-colonial breeding species were estimated by direct counts during the visits in the breeding season. To avoid adverse effects of ground census on the reproductive success of birds, colonies of cormorants, herons and egrets were located estanques salinos hechos por el hombre, de la salinera Exportadora de Sal, S.A. de C.V. (ESSA), las cuales no fueron incluidas en el estudio (fig. 1). Descripciones más amplias de las lagunas se pueden encontrar en Lewis y Ebeling (1974) y Saunders y Saunders (1981).

Entre finales de diciembre de 1997 y junio de 1998, realizamos siete inventarios a pie, en lanchas y en automóviles en ambas lagunas e inmediaciones para localizar los sitios de anidamiento de las aves acuáticas (incluyendo estructuras hechas por el hombre localizadas tierra adentro, adecuadas como sitios de 
early in the nesting season and delimited on a map. Later on, at the peak of the breeding season, pair numbers were estimated by visual counts using binoculars and a spotting scope from vantage points outside the colonies. Tern colonies were surveyed at the end of the breeding season to have a direct nest count. These estimates provide an approximate guide to the actual number of breeding pairs present. However, because of dense and mixed colony nesting, bird numbers are probably underestimated. This may be true for the sparse American oystercatcher (Haematopus palliatus). We include data gathered in surveys and inventories during the 1970s and 1980s by the staff of the Migratory Waterbird Refuge at Sebastián Vizcaíno Bay, an office of the federal government that managed the refuge between 1972 and 1988. We made comparisons with Bancroft (1927) and Massey and Palacios (1994) to find discrepancies and similarities of species composition and bird numbers.

A Landsat-based thematic digital image (DUMAC, 1995) of the coverage of the open waters and wetlands of the region, classified according to Cowardin et al. (1979), and a computer program Arc/Info PC-7 were used to estimate current natural and man-related modified wetland and open water coverages. Governmental protection programs implemented in the region were analyzed to clarify their role in the conservation of breeding waterbirds.

\section{RESULTS AND DISCUSSION}

\section{Species account}

\section{Larus occidentalis (western gull)}

Bancroft (1927) reported this gull on some of the islands of Scammon's Lagoon, but gave no figures on the number of breeding pairs. anidamiento de rapaces). Cada inventario comprendió cuatro días y fue realizado por tres observadores. Registramos datos sobre la presencia de las especies y de los sitios de anidamiento, así como del contenido de los nidos cuando fue posible. En un mapa de escala 1:50,000 se ubicaron los sitios de anidamiento y se estimó el número de parejas reproductoras.

El tamaño de población de las especies reproductoras no coloniales fue estimado por conteos directos en las visitas durante la estación reproductora. Para evitar el efecto adverso de los censos terrestres sobre el éxito reproductivo de las aves, las colonias de cormoranes y garzas se localizaron al inicio de la estación reproductora y se delimitaron en un mapa. Posteriormente, en el pico de la estación reproductora, se estimó el número de parejas mediante conteos visuales con el uso de binoculares y de un telescopio desde puntos elevados fuera de las colonias. Las colonias de las golondrinas marinas fueron inventariadas al final de la estación reproductora para tener un conteo directo de nidos. Estas estimaciones proveen de una guía aproximada del número de parejas reproductoras realmente presentes. Sin embargo, a causa del anidamiento colonial mixto y de la densidad de las colonias, los números de aves probablemente están subestimados. Esto también puede ser el caso del ostrero americano (Haematopus palliatus), muy disperso en su distribución. Nosotros incluimos datos obtenidos en exploraciones e inventarios durante los años setenta y ochenta por el personal del Refugio de Aves Acuáticas Migratorias en Bahía de Sebastián Vizcaíno, dependencia del gobierno federal que administró el refugio entre 1972 y 1988 . Hicimos comparaciones con los datos de Bancroft (1927) y Massey y Palacios (1994) para encontrar discrepancias y similitudes en la composición de especies y en el número de aves. 
K. Kenyon (1946, unpublished field notes) found numerous nesting pairs on Concha and Zacatosa islands. R. Osorio (1976, 1977, unpublished) found about 300 pairs on Piedra, Concha and Zacatosa islands, and reported total nest failures in 1976 on Piedra and Zacatosa islands caused by coyote (Canis latrans) depredation and tidal flooding. A. Castellanos (1980, pers. obs.) found 200 pairs nesting on Concha Island, as well as tidal flooding. Massey and Palacios (1994) reported recent breeding (nesting after 1984) at Scammon's Lagoon. In 1998, we located nests on Concha and Piedra islands.

\section{Sterna caspia (Caspian tern)}

Bancroft (1927) reported breeding in a colony very close to a royal tern (Sterna maxima) colony on an island. He reported that the Caspian terns "were not at all common", attributing the small numbers to the early date (27 May 1926) of his visit. Osorio (1976, unpublished) reported two colonies on Concha Island, very close to each other. According to his report, one colony was roughly estimated as 1500 pairs and the other numbered 200 nests. Everett and Anderson (1991) reported an active colony (about 20 pairs) in 1975. Massey and Palacios (1994) reported recent breeding at Scammon's Lagoon. We found a colony on Concha Island, close to a royal tern colony.

\section{Sterna maxima (royal tern)}

Bancroft (1927) found this species nesting mixed with elegant terns (Sterna elegans) on one island in Scammon's Lagoon, estimating "thousands" of birds. Kenyon (1946, unpublished field notes) reported nesting colonies, about 150 pairs, on Concha and Zacatosa islands, and consistent egg robbery by fishermen. Castellanos (1980, unpublished) reported
Se utilizó una imagen digital Landsat TM (DUMAC, 1995) de la cobertura de humedales y aguas abiertas de la región, tipificada de acuerdo con Cowardin et al. (1979), y el programa de computadora Arc/Info PC-7 para estimar las coberturas actuales del humedal natural y de las modificaciones relacionadas con el hombre. Se analizaron los programas de protección gubernamental instrumentados en la región para aclarar su efectividad en la conservación de las aves acuáticas reproductoras.

\section{RESULTADOS Y DISCUSIÓN}

\section{Recuento de especies}

Larus occidentalis (gaviota occidental)

Bancroft (1927) reportó esta gaviota en algunas de las islas de la Laguna Ojo de Liebre; sin embargo, no proporcionó ningún dato sobre el número de parejas anidando. K. Kenyon (1946, notas de campo no publicadas) encontró numerosas parejas anidando en las islas Concha y Zacatosa. R. Osorio (1976, 1977, no publicado) encontró aproximadamente 300 parejas en las islas Piedra, Concha y Zacatosa, y reportó el fracaso total de la anidación en 1976 en las islas Piedra y Zacatosa, causado por la depredación por coyotes (Canis latrans) y por marejadas. A. Castellanos (1980, obs. pers.) encontró 200 parejas anidando en la Isla Concha, colonia que fue inundada por las mareas. Massey y Palacios (1994) reportaron anidamiento reciente (posterior a 1984) en la Laguna Ojo de Liebre. En 1998 nosotros localizamos nidos en las islas Piedra y Concha.

\section{Sterna caspia (golondrina marina)}

Bancroft (1927) reportó anidamiento adyacente a una colonia de la golondrina marina real (Sterna maxima) en una isla. Él consideró que "no eran del todo comunes", atribuyendo 
about 400 pairs nesting on Concha Island and the destruction of this colony by tidal flooding. Everett and Anderson (1991), and Massey and Palacios (1994) reported recent breeding at Scammon's Lagoon. In 1998, we found this species nesting only on Concha Island, close to a Caspian tern colony. Based on photos by Bancroft (1927), showing beds of pecten shell (Argopecten ventricosus), we assume that it was on Concha Island (Shell Island) where he found this species in 1926. This island is the only place in the area that has a substrate of this type.

\section{Sterna elegans (elegant tern)}

Bancroft (1927) found a small number of eggs "intermingled with those of royal tern", according to photos in his report, probably of Concha Island. Kenyon (1946, unpublished field notes) exhaustively explored Concha Island; however, he did not positively confirm nesting of this species on the island. Everett and Anderson (1991), and Massey and Palacios (1994) listed the species as breeding at Scammon's Lagoon, but no figures of nesting pairs were provided. We were unable to find any nesting pairs; however, we cannot dismiss it as a breeding bird, because we saw some pairs over Concha Island flying near the royal and Caspian tern colonies.

Ardea herodias (great blue heron)

In 1926 there were few nesting pairs of this species and only in the marshes of Scammon's Lagoon (Bancroft, 1927). Osorio (1977, unpublished) did not report nesting of this species. F. Heredia (pers. comm.) found great blue herons nesting on Concha and Piedra islands since 1984. Massey and Palacios (1994) reported recent breeding in Scammon's Lagoon. In 1998, we found several pairs nesting on Piedra and Concha islands. su escaso número a la fecha temprana (27 de mayo de 1926) de su visita. Osorio (1976, no publicado) reportó dos colonias en la Isla Concha, muy cercana una de la otra. De acuerdo con su reporte, una colonia fue gruesamente estimada en 1500 parejas y la otra en 200 nidos. Everett y Anderson (1991) reportan una colonia activa (cerca de 20 parejas) en 1975. Massey y Palacios (1994) registraron anidamiento reciente en la Laguna Ojo de Liebre. Nosotros encontramos una colonia en Isla Concha muy cercana a un área de anidación de la golondrina marina real.

Sterna maxima (golondrina marina real)

Bancroft (1927) encontró esta especie anidando mezclada con la golondrina narina elegante (Sterna elegans) en una isla en la Laguna Ojo de Liebre, estimando "miles" de aves. Kenyon (1946, notas de campo no publicadas) reportó colonias reproductoras, cerca de 150 parejas, en las islas Concha y Zacatosa, y consistente saqueo de huevos por pescadores. Castellanos (1980, no publicado) encontró aproximadamente 400 parejas anidando en la Isla Concha y la destrucción de esta colonia por inundaciones. Everett y Anderson (1991) y Massey y Palacios (1994) reportaron anidamiento reciente en la Laguna Ojo de Liebre. En 1998, nosotros encontramos a esta especie anidando sólo en la Isla Concha cerca de una colonia de la golondrina marina cáspica. Con base en una foto de Brancroft (1927) que muestra un denso manto de almeja catarina (Argopecten ventricosus), suponemos que fue en la Isla Concha donde él encontró esta especie en 1926. Esta isla es el único lugar en la zona con un substrato de ese tipo.

Sterna elegans (golondrina marina elegante)

Bancroft (1927) encontró algunos huevos de esta especie "mezclados con aquellos de la 


\section{Phalacrocorax auritus (double-crested cormorant)}

Bancroft (1927) found several small colonies, about 80 occupied nests, at Scammon's Lagoon. Kenyon (1946, unpublished field notes) found several small colonies (5-12 pairs each) on three islands. He also observed egg robbery by fishermen. Osorio (1977, unpublished) found 980 nesting pairs on Concha and Zacatosa islands. Castellanos (1980, pers. obs.) counted 1100 occupied nests on Concha Island and some pairs on Zacatosa Island. SalinasZavala et al. (1991) found 560 pairs and 208 pairs nesting in 1984 on the islands of Piedra and Concha, respectively. Massey and Palacios (1994) reported nesting at Scammon's Lagoon. In 1998 we found several colonies on Piedra, Concha and Zacatosa islands.

Sterna antillarum (least tern)

Bancroft (1927) reported this species breeding at Scammon's Lagoon, based on a museum set of eggs collected on Zacatosa Island. Everett and Anderson (1991) reported nesting at Scammon's Lagoon, based on Massey (1977). Massey and Palacios (1994) reported that more than 60 pairs nested at Piedra Island and the man-made brine pan area. We were unable to find nesting evidence on Scammon's Lagoon during our surveys. According to E. Palacios (pers. comm.), some pairs also nest on Arena Island (the sand barrier at the mouth of Scammon's Lagoon).

Egretta thula (snowy egret)

Bancroft (1927) reported this species confined "to the marshes of two of the islands" at Scammon's Lagoon, but gave no figures on breeding pairs. Osorio (1977, unpublished) found four breeding pairs at Concha Island. Castellanos (pers. obs.) found six pairs in 1980 golondrina marina real", probablemente en la Isla Concha. Kenyon (1946, notas de campo no publicadas) exploró Isla Concha exhaustivamente; sin embargo, no confirmó positivamente anidamiento de esta especie en la isla. Everett y Anderson (1991) y Massey y Palacios (1994) la enlistan como anidante en la Laguna Ojo de Liebre, pero no proporcionan ningún número de parejas. Nosotros no pudimos encontrar parejas reproductoras; sin embargo, no la descartamos como una ave anidante, ya que vimos algunas parejas en la Isla Concha sobrevolando cerca de las colonias de las golondrinas marina real y cáspica.

\section{Ardea herodias (garzón azul)}

En 1926 había muy pocas parejas anidantes de esta especie y solamente en las marismas de la Laguna Ojo de Liebre (Brancroft, 1927). Osorio (1977, no publicado) no registró el anidamiento de esta especie. F. Heredia (com. pers.) encontró garzones azules anidando en las islas Concha y Piedra desde 1984. Massey y Palacios (1994) reportaron anidamiento reciente en la Laguna Ojo de Liebre. En 1998, nosotros encontramos varias parejas anidando en las islas Piedra y Concha.

Phalacrocorax auritus (cormorán de doble cresta)

Bancroft (1927) registró varias colonias pequeñas, cerca de 80 nidos ocupados, en la Laguna Ojo de Liebre. Kenyon (1946, notas de campo no publicadas) encontró varias colonias pequeñas ( 5 a 12 parejas en cada una) en tres islas. El observó que los nidos de estas colonias eran saqueados por los pescadores. Osorio (1977, no publicado) encontró 980 parejas reproductoras en las islas Concha y Zacatosa. Castellanos (1980, obs. pers.) contó 1100 nidos ocupados en la Isla Concha y algunas parejas en la Isla Zacatosa. Salinas-Zavala et al. (1991) 
on the same island. Massey and Palacios (1994) reported recent breeding at Scammon's Lagoon. We found breeding pairs on Concha and Piedra islands.

\section{Egretta rufescens (reddish egret)}

Bancroft (1927) reported this species breeding in marshes and on open beaches at Scammon's Lagoon. One of Bancroft's pictures shows two reddish egret nests probably on Concha Island. Kenyon (1946, unpublished field notes) found about 60-70 nesting pairs on one unidentified island. Osorio (1977, unpublished) found small colonies (70 nests) on Concha Island. Castellanos (1980, pers. obs.) also found 213 nests there. Salinas-Zavala et al. (1991) reported about 35 pairs nesting on Concha Island. Massey and Palacios (1994) reported recent breeding at Scammon's Lagoon. We found nests only on Concha and Piedra islands.

\section{Egretta alba (great egret)}

Bancroft (1927) gives no report of this species at Scammon's Lagoon. Wilbur (1987) listed it as an "uncommon and sporadic visitor" of Baja California with "no certain breeding records". No records are available from the 1970s and 1980s. Everett and Anderson (1991) did not report nesting of this species in the area. Massey and Palacios (1994), however, reported recent breeding (three breeding pairs) at Scammon's Lagoon. We found several pairs nesting only on Concha Island.

\section{Egretta tricolor (Louisiana heron)}

Bancroft (1927) reported this species as "a marsh breeder" at Scammon's Lagoon. Osorio (1976, unpublished) reported 24 pairs nesting on Concha Island. Massey and Palacios (1994) reported recent breeding at Scammon's Lagoon encontraron 560 y 208 parejas reproductoras en 1984 en las islas Piedra y Concha. Massey y Palacios (1994) reportaron anidamiento en la Laguna Ojo de Liebre. En 1998 encontramos varias colonias en las islas Piedra, Concha y Zacatosa.

Sterna antillarum (charrán mínimo)

Bancroft (1927) reportó esta especie anidando en la Laguna Ojo de Liebre, con base en un juego de huevos de un museo recolectado en la Isla Zacatosa. Everett y Anderson (1991) registraron anidamiento en la Laguna Ojo de Liebre, con base en Massey (1977). Massey y Palacios (1994) reportaron que más de 60 parejas anidan en el área de estanques salinos y en la Isla Piedra. Nosotros no pudimos encontrar evidencias de anidamiento en los humedales naturales de la Laguna Ojo de Liebre durante nuestros inventarios. De acuerdo con E. Palacios (com. pers.), algunas parejas anidan también en la Isla Arena.

\section{Egretta thula (garcita blanca)}

Bancroft (1927) reportó esta especie confinada "a las marismas de dos de las islas" en la Laguna Ojo de Liebre, pero no dio números de parejas anidantes. Osorio (1977, no publicado) encontró cuatro parejas anidando en la Isla Concha. Castellanos (obs. pers.) encontró seis parejas en 1980 en la misma isla. Massey y Palacios (1994) reportaron anidamiento reciente en la Laguna Ojo de Liebre. Nosotros encontramos parejas reproductoras en las islas Concha y Piedra.

\section{Egretta rufescens (garza cabeza roja)}

Bancroft (1927) reportó esta especie anidando en marismas y playas abiertas en la Laguna Ojo de Liebre. Una de las fotos de 
but no specific location. We found this species only on Concha Island.

Nycticorax violaceus (yellow-crowned night heron)

Bancroft (1927) defined this bird "as the rarest of the larger breeding waterbirds we found in Scammon's". He found several pairs nesting "on the marshes". Massey and Palacios (1994) reported recent breeding in Scammon's Lagoon, but they did not provide any numbers and specific locations. We found several pairs nesting only on Piedra Island.

\section{Nycticorax nycticorax (black-crowned night heron)}

Bancroft (1927) found this species nesting (about 100 pairs) exclusively in bushes on the beach at Scammon's Lagoon. Osorio (1977, unpublished) reported nesting only on Concha Island. He gave no total number of breeders, though he monitored 55 nests during the breeding season. Castellanos (pers. obs.) found 56 pairs on the same island in 1980. SalinasZavala et al. (1991) reported four nests on this island in 1984. Massey and Palacios (1994) reported recent breeding at Scammon's Lagoon. We found many nesting pairs on Concha and Piedra islands.

Falco peregrinus (peregrine falcon)

Before 1994, there were no nesting records of this species for Scammon's Lagoon. Massey and Palacios (1994) reported recent breeding, estimating six pairs nesting in the area. Castellanos et al. (1994a) reported three breeding pairs, suggesting this species has nested in the area since at least the 1970s. In 1998 we found eight pairs; four of these had laid eggs: one on Piedra Island, two on channel markers in the El Chaparrito channel, and one
Bancroft muestra dos nidos de garza cabeza roja, probablemente en la Isla Concha. Kenyon (1946, notas de campo no publicadas) encontró 60 a 70 parejas en una isla no identificada. Osorio (1977, no publicado) encontró pequeñas colonias (70 nidos) en la Isla Concha. Castellanos (1980, obs. pers.) encontró 213 nidos ahí mismo. Salinas-Zavala et al. (1991) reportaron cerca de 35 parejas anidando en la Isla Concha. Massey y Palacios (1994) reportaron anidamiento reciente en la Laguna Ojo de Liebre. Nosotros encontramos nidos sólo en las islas Concha y Piedra.

Egretta alba (garzón blanco)

Bancroft (1927) no dio ningún reporte de esta especie en la Laguna Ojo de Liebre. Wilbur (1987) la reportó como un "visitante no común y esporádico" de Baja California con "registros inciertos de anidamiento". No existen registros de los años setenta y ochenta. Everett y Anderson (1991) no reportan anidamiento de esta especie en el área. Massey y Palacios (1994) reportaron anidamiento reciente y tres parejas en la Laguna Ojo de Liebre. Nosotros encontramos varias parejas anidando sólo en la Isla Concha.

\section{Egretta tricolor (garza flaca)}

Bancroft (1927) reportó esta especie “como un anidante de las marismas" en la Laguna Ojo de Liebre. Osorio (1976, no publicado) reportó 24 parejas anidando en la Isla Concha. Massey y Palacios (1994) reportaron anidamiento reciente en la Laguna Ojo de Liebre sin especificar sitio. Nosotros la encontramos sólo en la Isla Concha.

Nycticorax violaceus (pedrete enmascarado)

Bancroft (1927) definió a esta ave "como la más rara de las aves acuáticas reproductoras 
on a nesting platform built by ESSA at Scammon's Lagoon. In some years, a nesting pair has been observed on Cholla Island (Palacios, pers. comm.).

\section{Haematopus palliatus (American} oystercatcher)

Bancroft (1927) described Scammon's Lagoon as "a haven for oystercatchers" and estimated that at least $100-150$ pairs were distributed on the islands, but he did not give nest numbers or specific locations. Kenyon (1946, unpublished field notes) observed about 39 pairs, but only a small number of active nests, on four islands. He reported consistent egg robbery by fishermen. Osorio (1977, unpublished) found 16 pairs on Concha and Zacatosa islands. Massey and Palacios (1994) reported recent breeding at Scammon's Lagoon. We saw many pairs on the islands and beaches, but we found seven active nests only on Concha and Piedra islands. The number of pairs seems to be lower than those reported by Bancroft (1927).

\section{Larus atricilla (laughing gull)}

No historic report of nesting of this species exists at Scammon's Lagoon prior to 1994 (Castellanos et al., 1994b). In that year, four pairs nested on Isla Piedra. In 1998 this species was nesting only on the same island.

\section{Pandion haliaetus (osprey)}

Bancroft (1927) found ospreys confined to the islands in Scammon's Lagoon. Kenyon (1947) found 27 active pairs, and reported the robbery of eggs and young by fishermen. Between the late 1960s and early 1970s, some pairs occupied channel markers outside the islands as nesting sites (Henny and Anderson, 1979). In the early 1980s, ospreys began to nest inland on utility poles at Guerrero Negro. In grandes que encontramos en la Laguna Ojo de Liebre". Él encontró varias parejas anidando en las marismas. Massey y Palacios (1994) reportaron anidamiento reciente en la Laguna Ojo de Liebre, pero no proporcionaron ningún número de parejas ni localidades específicas. Nosotros encontramos varias parejas anidando sólo en la Isla Piedra.

Nycticorax nycticorax (perro de agua)

Bancroft (1927) encontró cerca de 100 parejas de esta especie anidando exclusivamente sobre arbustos en las playas de la Laguna Ojo de Liebre. Osorio (1977, no publicado) reportó anidamiento sólo en la Isla Concha. Él no dio un número total de parejas; sin embargo, monitoreó 55 nidos durante la temporada reproductora. Castellanos (obs. pers.) encontró 56 parejas en la misma isla en 1980. Salinas-Zavala et al. (1991) encontraron cuatro nidos en la Isla Concha en 1984. Massey y Palacios (1994) reportaron anidamiento reciente en la Laguna Ojo de Liebre. Nosotros encontramos muchas parejas anidando en las islas Piedra y Concha.

\section{Falco peregrinus (halcón peregrino)}

Antes de 1994, no había registros de anidamiento de esta especie en la Laguna Ojo de Liebre. Massey y Palacios (1994) reportaron anidamiento reciente estimando que seis parejas anidaron en el área. Castellanos et al. (1994a) reportaron tres parejas, sugiriendo que esta ave anida en el área desde los años setenta. En 1998, encontramos ocho parejas; cuatro de éstas pusieron huevos: una en la Isla Piedra, dos en balizas en el canal de navegación El Chaparrito y una en una plataforma de anidamiento construida por ESSA en la Laguna Ojo de Liebre. En algunos años, se ha observado una pareja anidando en la Isla Cholla (Palacios, com. pers.). 
1982, 86 pairs nested in the study area, nearly $30 \%$ on artificial nesting sites. In 1993, 126 pairs nested at both lagoons and the mainland, about $50 \%$ on artificial nesting sites (see Castellanos and Ortega-Rubio, 1995). In 1998 we counted 152 pairs occupying the islands, channel markers, nest platforms, utility poles, and other man-made structures in the urban landscape.

\section{Changes in species presence, distribution and population size}

Since 1926, some changes of the species composition of breeding waterbirds have occurred in the study area. Of 17 species reported by Bancroft (1927), we found 12 breeding on the small islands of Scammon's Lagoon. Four (least tern, Sterna antillarum; snowy plover, Charadrius alexandrinus; Wilson's plover, Charadrius wilsonia; and clapper rail, Rallus longirostris) were not detected nesting on the lagoon islands or salt marshes during our surveys. However, least terns and Wilson's plovers breed on Piedra Island (Massey and Palacios, 1994), and snowy plovers on Arena Island, in saline flatlands near Guerrero Negro and in the man-made brine pan area (Danemann and Carmona, 2000) (table 1). The clapper rail, reported by Bancroft (1927) nesting on the salt marsh fringe of one unidentified island, and Wilson's plover have been detected nesting recently at the salt marshes of Scammon's Lagoon (Palacios, pers. comm.). The elegant tern is probably still nesting on Concha Island. Three species have become new residents: the great egret (Massey and Palacios 1994), the peregrine falcon (Castellanos et al., 1994a), and the laughing gull (Castellanos et al., 1994b).

According to Bancroft (1927), there were four species (tricolored heron, reddish egret, black-crowned night heron and yellowcrowned night heron) at few sites and in small
Haematopus palliatus (ostrero americano)

Bancroft (1927) describió a la Laguna Ojo de Liebre como "un paraíso para los ostreros" y estimó que al menos 100 ó 150 parejas estaban distribuidas en las islas, pero no proporcionó número de nidos ni localidades específicas. Kenyon (1946, notas de campo no publicadas) observó aproximadamente 39 parejas pero sólo un muy pequeño número de nidos activos, en cuatro islas. Él reportó consistente saqueo de huevos por pescadores. Osorio (1977, no publicado) encontró 16 parejas en las islas Concha y Zacatosa. Massey y Palacios (1994) reportaron anidamiento reciente en la Laguna Ojo de Liebre. Nosotros observamos muchas parejas en las islas y playas; sin embargo, sólo encontramos siete nidos con huevos en las islas Concha y Piedra. Los números de parejas parecen estar por debajo de los reportados por Bancroft (1927).

\section{Larus atricilla (gaviota reidora)}

Antes de 1994 no existía ningún reporte histórico de anidamiento de esta especie en la Laguna Ojo de Liebre (Castellanos et al., 1994b). En ese año cuatro parejas anidaron en la Isla Piedra. En 1998 esta especie estaba anidando de nuevo en Isla Piedra.

\section{Pandion haliaetus (águila pescadora)}

Bancroft (1927) encontró a las águilas pescadoras confinadas a las islas de la Laguna Ojo de Liebre. Kenyon (1947) encontró 27 parejas activas y reportó saqueo de huevos y pollos por pescadores. Entre finales de los años sesenta y principios de los setenta, algunas parejas ocuparon balizas fuera de las islas como sitios de anidamiento (Henny y Anderson, 1979). A principios de los ochenta, las águilas pescadoras comenzaron a anidar tierra adentro en los postes de los tendidos 
Table 1. Breeding waterbirds at Ojo de Liebre and Guerrero Negro lagoons. $\mathrm{P}=$ Piedra Island; $\mathrm{C}=$ Concha Island; $\mathrm{Z}=$ Zacatosa Island; $\mathrm{A}=$ Alambre Island; $\mathrm{HL}=$ humanized landscape; $\mathrm{NS}=$ not seen; $\mathrm{BNNR}=$ breeding but nest numbers not reported; NOM-059 = Norma Oficial Mexicana.

Tabla 1. Avifauna reproductora en las lagunas Ojo de Liebre y Guerrero Negro. $\mathrm{P}=$ Isla Piedra; $\mathrm{C}=$ Isla Concha; $\mathrm{Z}=$ Isla Zacatosa; $\mathrm{A}=$ Isla Alambre; $\mathrm{HL}=$ paisaje humanizado; $\mathrm{NS}=$ no visto; $\mathrm{BNNR}=$ anidando pero números de nidos no reportados; NOM-059 = Norma Oficial Mexicana;

\begin{tabular}{|c|c|c|c|c|c|}
\hline Species & Bancroft (1927) & $\begin{array}{c}\text { Massey and } \\
\text { Palacios } \\
\text { (1994) pairs }\end{array}$ & $\begin{array}{c}\text { This study } \\
\text { pairs }\end{array}$ & $\begin{array}{l}1998 \text { nesting } \\
\text { spots within } \\
\text { the lagoons }\end{array}$ & $\begin{array}{l}\text { Conserva- } \\
\text { tion status } \\
\text { NOM-059 }\end{array}$ \\
\hline Phalacrocorax auritus & 80 pairs & 3500 & 2500 & $\mathrm{P}, \mathrm{C}, \mathrm{Z}$ & Rare \\
\hline Ardea herodias & Very few pairs & 5 & 60 & $\mathrm{P}, \mathrm{C}$ & \\
\hline Egretta alba & NS & 3 & 10 & $\mathrm{C}$ & \\
\hline Egretta thula & BNNR & 20 & 55 & $\mathrm{P}, \mathrm{C}$ & \\
\hline Egretta tricolor & 75 pairs & 17 & 7 & $\mathrm{C}$ & \\
\hline Egretta rufescens & BNNR & 50 & 580 & $\mathrm{P}, \mathrm{C}$ & Threatened \\
\hline Nycticorax nycticorax & 100 pairs & 50 & 95 & $\mathrm{P}, \mathrm{C}$ & \\
\hline Nycticorax violaceus & The rarest & NS & 12 & $\mathrm{P}$ & \\
\hline Pandion haliaetus & BNNR & 76 & 152 & $\begin{array}{l}\mathrm{P}, \mathrm{C}, \mathrm{Z}, \\
\mathrm{A}, \mathrm{HL}\end{array}$ & \\
\hline Falco peregrinus & NS & 6 & 8 & $\mathrm{P}, \mathrm{HL}$ & Threatened \\
\hline Rallus longirostris & 4 adults & NS & NS & & \\
\hline Charadrius alexandrinus & Many chicks & $190^{*}$ & NS & & \\
\hline Charadrius wilsonia & 1 broken egg & BNNR & NS & & \\
\hline Haematopus palliatus & $100-150$ pairs & 28 & 77 & $\mathrm{P}, \mathrm{C}$ & \\
\hline Larus atricilla & NS & NS & 4 & $\mathrm{P}$ & \\
\hline Larus occidentalis & BNNR & 355 & 450 & $\mathrm{P}, \mathrm{C}$ & \\
\hline Sterna caspia & Not all common & 160 & 1142 & $\mathrm{C}$ & \\
\hline Sterna maxima & $\begin{array}{l}\text { Thousands of } \\
\text { birds }\end{array}$ & 500 & 2762 & $\mathrm{C}$ & \\
\hline Sterna elegans & Half-dozen eggs & NS & NS & & Threatened \\
\hline Sterna antillarum & $\begin{array}{l}\text { NS. Reported } \\
\text { on a set of } \\
\text { museum eggs }\end{array}$ & $60 * *$ & NS & & Endangered \\
\hline
\end{tabular}

$*=$ Found at the saltwork area; $* *=$ found at the saltwork area and Piedra Island. 
numbers (1-12 individuals), nesting on the salt marshes outside the islands. By 1946, Kenyon (unpublished field notes) did not report any species nesting on the salt marshes. Except for the reddish egret nesting colony on one of the islands, he found very small numbers of herons and egrets on the other islands, but not nests. This suggests that the abandonment of salt marshes as nesting sites occurred before the establishment of the ESSA saltworks in the area. Despite these changes, actual nesting habitat arrangement within Scammon's Lagoon and islands is similar to that found by Bancroft (1927); most of the waterbird species and the bulk of numbers still breed on the small islands. In addition to these changes in composition of the nesting species of the lagoons, Danemann and Carmona (2000) reported eight species nesting at the ESSA saltworks brine pans; two of these species (Rynchops niger and Sterna nilotica) are new breeders in the region.

Because of the limited historical data, population changes cannot be traced for all species. However, we can have some insight of the changes based on data from 1970 and 1980, and the Massey and Palacios (1994) records (table 1). Data show that most of the species and pair numbers remained confined to the small islands. However, some species have benefited from the human-induced changes, occupying suitable nesting sites in the urban landscape (osprey), man-made brine pans (least tern, snowy plover, American oystercatcher, Caspian tern and royal tern) (see Massey and Palacios, 1994; Danemann and Carmona, 2000), and artificial sites such as utility poles, towers and channel markers (osprey and peregrine falcon). When compared to figures provided by Massey and Palacios (1994), we find some discrepancies. However, we believe this does not reflect recent changes in population size but in estimating procedures. Our data suggest that most species increased their eléctricos de Guerrero Negro. En 1982, 86 parejas anidaron en el área de estudio, cerca del $30 \%$ en sitios de anidamiento artificiales. En 1993, 126 parejas anidaron en ambas lagunas y tierra adentro, aproximadamente $50 \%$ sobre sitios artificiales de anidamiento (Castellanos y Ortega-Rubio, 1995). En 1998, nosotros contamos 152 parejas ocupando las islas, balizas en canales de navegación, plataformas de anidamiento, postes de la red eléctrica y otras estructuras antropogénicas en el paisaje urbano.

\section{Cambios en la presencia de especies, distribución y tamaños de población}

Desde 1926, han ocurrido algunos cambios en la composición de especies de aves acuáticas reproductoras del área de estudio. De 17 especies reportadas por Bancroft (1927), nosotros encontramos 12 anidando en las islas de la Laguna Ojo de Liebre. Cuatro (Sterna antillarum, charrán mínimo; Charadrius alexandrinus, chorlito níveo; Charadrius wilsonia, chorlito piquigrueso; y Rallus longirostris, gallina de agua) no fueron detectadas anidando en las marismas o en las islas durante nuestros inventarios. Sin embargo, el charrán mínimo y el chorlito piquigrueso han anidado recientemente en la Isla Piedra (Massey y Palacios, 1994), y el chorlito níveo en la Isla Arena, en salitrales cercanos a Guerrero Negro (Massey y Palacios, 1994) y en los estanques salinos hechos por el hombre (Danemann y Carmona, 2000) (tabla 1). La gallina de agua, reportada por Bancroft (1927) anidando en la marisma adyacente a una isla no identificada, y el chorlito piquigrueso han sido detectados anidando recientemente en las marismas de la Laguna Ojo de Liebre (Palacios, com. pers.). La golondrina marina elegante probablemente todavía anida en la Isla Concha. Tres especies son nuevos residentes de la laguna: el garzón blanco (Massey y Palacios, 
numbers since 1926. The species less abundant now are the American oystercatcher and tricolored heron.

\section{Problems and threats}

\section{Habitat changes}

Before 1946, the area was pristine (see Bancroft, 1927). By 1946 a small number of fishermen inhabited some of the islands (Kenyon, 1946 unpublished field notes, 1947). Scammon's Lagoon, surrounded by about 123,000 ha of hypersaline intertidal flatlands (salitrales) remained in such condition until 1953, when a saltworks was established and a small town, Guerrero Negro, settled in the lagoon's vicinity. Between 1957 and 1968, about 12,000 ha of the flatlands were flooded and transformed into production salt ponds (L. Villavicencio, pers. comm.). By the mid-1970s, the flooded surface was about 32,000 ha (J.C. Peralta, pers. comm.), increasing between the late $1970 \mathrm{~s}$ and 1987 to its current size, about 37,500 ha. However, about 160 ha $(0.25 \%)$ of the presettlement natural salt marshes and open waters at Scammon's and Guerrero Negro lagoons were reclaimed by the saltworks development. Such changes had no significant impact on the composition, structure and function of the natural wetland complex. Most of the changes occurred in the intertidal flatlands, a hypersaline and impoverished wildlife habitat with limited value for waterbirds, adjacent to Scammon's Lagoon. About $30 \%$ of its surface was flooded, creating a saline wetland with suitable habitats for waterbirds (see Carmona and Danemann, 1998; Danemann and Carmona, 2000) (fig. 1).

Current natural nesting habitats, restricted to the small islands, seem to be critical in the area. Most of the birds nest on Piedra and Concha islands. The other islands apparently
1994), el halcón peregrino (Castellanos et al., 1994a) y la gaviota reidora (Castellanos et al., 1994b).

De acuerdo con Bancroft (1927), había cuatro especies (garza flaca, garza cabeza roja, perro de agua y pedrete enmascarado) en pocos sitios y en escasos números (de 1 a 12 individuos) anidando en las marismas fuera de las islas. En 1946, Kenyon (notas de campo no publicadas) no reportó ninguna especie anidando en las marismas y excluyendo una colonia de garza cabeza roja de una isla no identificada, encontró muy pocos individuos de otras garzas, ninguno anidando, en las islas. Esto sugiere que el abandono de las marismas como sitios de anidamiento sucedió antes de que en la zona se estableciera la salinera de ESSA. A pesar de estos cambios, el arreglo actual de los sitios de anidamiento dentro del humedal natural y las islas es similar a aquel que encontró Bancroft (1927); la mayoría de las especies de aves acuáticas y de los individuos anidan en las islas. Además de estos cambios en la composición de especies que anidan en la laguna, Danemann y Carmona (2000) reportaron ocho especies anidando en los estanques de producción de sal de ESSA; dos de ellas (Rynchops niger y Sterna nilotica) son nuevos reproductores en la región.

Debido a los escasos datos históricos, los cambios de población no pueden ser trazados para todas las especies. Sin embargo, podemos tener alguna idea de los cambios con base en los registros de los años setenta y ochenta y de Massey y Palacios (1994) (tabla 1). Los datos muestran que la mayoría de las especies permanecieron confinadas a las islas. Sin embargo, algunas especies han sido favorecidas por los cambios inducidos por el hombre sobre el hábitat, ocupando sitios de anidamiento adecuados en el paisaje urbano (águila pescadora), en los estanques salinos hechos por el hombre (charrán mínimo, chorlito níveo, ostrero, golondrina cáspica y golondrina marina real) 
lack suitable substrates for colonial nesting birds and are vulnerable to land predators or tidal flooding, supporting only a small number of cormorants and ospreys. Concha Island has lost about $35 \%$ of its size since 1984 , due to wave erosion. Most of the former nesting colonies there moved to other areas. Tern colonies still remain there, probably because it is the only island that has the nesting substrate they require.

Predation

Cholla, Piedra and Alambre islands are accessible to land predators. Coyote predation precluded osprey nesting for several years before 1970 on Piedra and Alambre islands (Kenyon, 1947; Jehl 1977; Henny and Anderson, 1979). In the late 1970s and early 1980s, coyotes frequently invaded Piedra and Alambre islands, causing the abandonment of waterbird colonies and nesting sites (Castellanos, 1983). In those years, Concha island was the main nesting spot in the area.

Human perturbation

Before 1972, the islands were used as temporary fishing camps; breeding colonies were disturbed, and nests and young of many species robbed (Kenyon, 1947; Castellanos, 1983). Nest robbery was very consistent and occurred to such an extent that breeding colonies of some species were extirpated from the islands (Kenyon 1946, unpublished field notes). However, law enforcement and education programs implemented since 1972 by the refuge and reserve staffs eliminated these practices. Currently, the two main activities causing disturbance (small boat traffic) in the lagoons are fishing and tourism. Although there is an official restriction on visiting the islands, some evidence (e.g., trash and fire remains) suggest they are still visited. (ver Massey y Palacios, 1994; Danemann y Carmona, 2000), y en sitios artificiales como postes del tendido eléctrico, torres y balizas (águila pescadora y halcón peregrino). Cuando se comparan con los datos de Massey y Palacios (1994), se encuentran algunas discrepancias; sin embargo, creemos que éstas no reflejan cambios recientes en los tamaños de población sino en los procedimientos de estimación. Nuestros datos sugieren que la mayoría de las especies incrementaron sus números desde 1926. Las especies que disminuyeron en número son el ostrero americano y la garza flaca.

\section{Problemas y amenazas}

Cambios en el hábitat

Antes de 1946, el área era prístina (ver Bancroft, 1927). En 1946 algunas de las islas estaban habitadas por un pequeño número de pescadores (Kenyon, 1946 notas de campo no publicadas, 1947). La Laguna Ojo de Liebre, rodeada de cerca de 123,000 ha de planicies hipersalinas (salitrales), permaneció en esa condición hasta 1953, cuando una salinera fue establecida y un pequeño poblado, Guerrero Negro, emergió en la vecindad de las lagunas. Entre 1957 y 1968, cerca de 12,000 ha de los salitrales fueron inundadas y transformadas en estanques de producción de sal (L. Villavicencio, com. pers.). A mediados de los setenta, la superficie de salitrales inundada era de cerca de 32,000 ha (J.C. Peralta, com. pers.), incrementándose entre finales de los setenta y 1987 a su tamaño actual de aproximadamente 37,500 ha. En total, en las lagunas Ojo de Liebre y Guerrero Negro se rellenaron o dragaron aproximadamente 160 ha $(0.25 \%)$ de las marismas naturales y aguas abiertas existentes antes de que se estableciera la salinera de ESSA. Tales cambios no han tenido un impacto significativo en la composición y función de 


\section{Conservation}

Resident waterbirds are granted legal protection through the wildlife refuge and the El Vizcaíno Biosphere Reserve. Both units seek, theoretically, to maximize species diversity and maintain remnants of natural habitats. Five species have protection status according to Mexican norms (table 1). However, staff and resources assigned to waterbird conservation have been scarce. The protection measures implemented are incipient, focused on habitat protection, law enforcement and targeted at single species. Restrictions on land use established in the decrees of the refuge and the biosphere reserve prevented permanent new settlements and development of productive activities on the coast and inside the lagoons, which has probably favored habitat protection. Also, the presence of refuge and reserve staff, their law-enforcement functions, environmental education campaigns, and the successful nesting sites management programs of osprey and peregrine falcon (see Castellanos and Ortega-Rubio, 1995; Castellanos et al., 1997), were essential to eradicate the robbing of nests, diminish direct human perturbation of colonies on the islands, and favored the appreciation of waterbirds by local residents (Ortega-Rubio et al., 1998). Despite these achievements, the conservation needs of the breeding waterbird community have not been integrally attended. Also, no plan for waterbird use exists, which ignores their potential contribution to the reserve objective of promoting sustainable use of their resources to benefit the local population.

\section{CONCLUSIONS AND RECOMMENDATIONS}

Despite the limited protection and the recent development of the area, the evidence analyzed shows that the breeding waterbird todo el complejo de humedales naturales. La mayor parte de los cambios de cobertura ocurrieron en las planicies intermareales adyacentes a la Laguna Ojo de Liebre, un hábitat hipersalino carente de vida silvestre con limitado valor para las aves acuáticas. Aproximadamente el $30 \%$ de su superficie fue inundada, creándose un humedal salino, con hábitats accesibles para las aves acuáticas (ver Carmona y Danemann, 1998; Danemann y Carmona, 2000) (fig. 1).

Los hábitats naturales de anidamiento actuales, restringidos a las pequeñas islas, parecen ser críticos en el área. La mayoría de las aves anidan en las islas Piedra y Concha. Las otras islas aparentemente carecen de substratos adecuados para el anidamiento colonial de las aves y son vulnerables a los depredadores terrestres o a las inundaciones por mareas, conteniendo un pequeño número de cormoranes $\mathrm{y}$ de águilas pescadoras. La Isla Concha ha perdido desde 1984 aproximadamente el 35\% de su tamaño debido a la erosión por el oleaje. La mayoría de sus antiguas colonias se movieron a otras áreas. Las colonias de golondrinas marinas todavía permanecen ahí, probablemente porque es la única isla que tiene el substrato de anidamiento que ellas requieren.

\section{Depredación}

Las islas Cholla, Piedra y Alambre son accesibles a los depredadores terrestres. La depredación por coyote impidió el anidamiento del águila pescadora por varios años antes de 1970 en las islas Piedra y Alambre (Kenyon, 1947; Jehl, 1977; Henny y Anderson, 1979). A finales de los setenta y principios de los ochenta, los coyotes frecuentemente invadieron las islas Piedra y Alambre, causando el abandono de las colonias y de los sitios de anidamiento de las aves acuáticas (Castellanos, 1983). En esos años, Isla Concha fue el sitio de concentración de nidos en el área. 
community is still well preserved, and that man-made induced changes have not had a significant impact on natural wetland coverage and nesting substrates historically available at Scammon's and Guerrero Negro lagoons. This area still has a high value as a nesting habitat for a large waterbird community.

We believe this has been favored by the following conditions: (i) the sparse population and isolation of the peninsula, which precludes much industrial development; (ii) salt production, the main productive activity, was developed not on the natural wetlands or open water of both lagoons, but in the hypersaline habitat nearby, and this activity has also increased the suitable habitat for waterbirds in the region (see Ortega-Rubio et al., 1998; Carmona and Danemann, 1998; Danemann and Carmona, 2000); (iii) restrictions to settlements and to major and intensive activities based on the refuge and reserve decrees and authorities' vision of protected areas as a means to protect nature values; and (iv) a de facto non-development zoning because the greater part of the lagoon's surrounding area belongs to the ESSA mine concession and is strictly under its control.

The evidence analyzed suggests that the few nesting sites originally located in marshes were abandoned, probably because of human perturbation after the area was settled in the mid-1940s. Nest robbery and small boat traffic may have played a major role in this. Birds may have shifted toward the islands. However, except for the clapper rail and elegant tern, the species found on the islands in 1926 still breed there, have stable populations or have increased in number. Some of these species expanded their nesting ranges beyond the islands.

Suitable natural nesting habitats are scarce for most colonial waterbirds and some substrates are disappearing because of wave erosion. Reoccupation of marshes by herons and

\section{Perturbación humana}

Antes de 1972, las islas fueron utilizadas como campamentos temporales de pesca, las colonias de anidamiento eran perturbadas y los huevos y pollos de algunas especies saqueados (Kenyon, 1947; Castellanos, 1983). El saqueo de nidos era de tal magnitud y consistencia que las colonias reproductoras de algunas especies fueron extirpadas de las islas (Kenyon, 1946, notas de campo no publicadas). Sin embargo, la vigilancia y los programas de educación ambiental instrumentados por el personal del refugio y la reserva eliminaron estas prácticas. Actualmente, las dos principales actividades que causan perturbación (tránsito de lanchas) en las lagunas son la pesca y el turismo. No obstante que hay restricción oficial para visitar las islas, algunas evidencias (e.g., basura y restos de fogatas) sugieren que éstas son todavía visitadas.

\section{Conservación}

Las aves acuáticas residentes obtuvieron protección legal mediante el establecimiento del refugio de fauna silvestre y la Reserva de la Biósfera El Vizcaíno. Ambas unidades, teóricamente, buscan mantener los remanentes de los hábitats naturales y maximizar la diversidad de especies. Cinco especies tienen un estatus de protección de acuerdo con las normas mexicanas (tabla 1). Sin embargo, el personal y los recursos asignados para la conservación de las aves acuáticas han sido escasos. Las medidas de protección instrumentadas han sido incipientes, enfocadas a la protección del hábitat, la vigilancia y el cuidado de algunas especies individuales. Las restricciones en el uso del suelo establecidas en los decretos de refugio y la reserva de la biósfera han impedido el establecimiento de asentamientos humanos permanentes y el desarrollo de otras actividades productivas en la costa y dentro de 
egrets as nesting sites at the lagoon does not appear feasible because of the current level of small boat traffic. Some waterbirds, however, began to nest within the man-made ponds and urban landscape, which provide alternative nesting habitats.

We believe the biosphere reserve status of the area may provide the strategic framework and the resources needed for the conservation of breeding waterbirds. We recommend that the following be considered in any conservation strategy for the area: (i) protect breeding waterbirds as an essential element of the regional biodiversity, in a multispecies approach, integrating their management into a single natural and man-made (the saltworks) wetland unit; (ii) provide immediate protection against predators of nesting sites on the islands of Piedra, Alambre and Cholla, by monitoring before and during the breeding season, the physical removal of predators and fencing of potential routes of access; (iii) control wave erosion and rehabilitate habitat loss on Concha Island (the machinery and engineering capabilities at ESSA exist for such a task; (iv) prevent human perturbation at nesting sites located on man-made structures, and in the urban and saltwork landscapes; (v) protect against wave erosion at the new nesting sites within the salt ponds and evaluate the feasibility of creating larger potential nesting islets; (vi) increase the public concern of these birds; (vii) investigate and develop mechanisms for breeding waterbirds to provide economic benefits for reserve residents; and (viii) promote consensual and participatory approaches between stakeholders (fishermen, tourism agencies, ESSA, farmers, ranchers) and the reserve authorities, for attaining conservation and development objectives based on breeding waterbirds.

\section{ACKNOWLEDGEMENTS}

We thank CONACYT for the financial support of this research, SEMARNAP and ESSA las lagunas, lo cual probablemente ha favorecido la protección del hábitat. Por otro lado, la presencia del personal del refugio y la reserva, su función de vigilancia, sus campañas de educación ambiental y los programas de manejo de sitios de anidamiento del águila pescadora y del halcón peregrino, que han sido exitosos (ver Castellanos y Ortega-Rubio, 1995; Castellanos et al., 1997), fueron esenciales para erradicar el saqueo de nidos, disminuir la perturbación directa de las colonias en las islas y favorecer el aprecio de las aves acuáticas por los pobladores locales (Ortega-Rubio et al., 1998). No obstante estos logros, las necesidades de conservación de la comunidad de aves acuáticas reproductoras no han sido atendidas integralmente. Asimismo, no existe un plan para el manejo y aprovechamiento de las aves acuáticas, lo cual deja a un lado su potencial contribución al objetivo de la reserva de promover el uso sustentable de sus recursos para beneficiar a la población local.

\section{CONCLUSIONES Y RECOMENDACIONES}

A pesar de la protección limitada y del reciente desarrollo del área, las evidencias analizadas muestran que la comunidad de aves acuáticas reproductoras está todavía bien conservada y que los cambios inducidos por el hombre no han tenido un impacto significativo en la cobertura de los humedales naturales, ni en los substratos de anidamiento históricamente disponibles en las lagunas Ojo de Liebre y Guerrero Negro. Esta área todavía tiene un alto valor como hábitat de anidamiento para una gran comunidad de aves acuáticas.

Nosotros creemos que esto ha sido favorecido por las siguientes condiciones: (i) la dispersa población y el aislamiento de la península que limita mucho el desarrollo industrial; (ii) la producción de sal, la principal actividad productiva, fue desarrollada principalmente no en los humedales naturales o en las aguas 
for their institutional support, and S.R. Osorio and E. Palacios for their unpublished data. We are grateful to E. Palacios and two anonymous reviewers for the critical reading of the manuscript. We also thank P. Galina, I. Tovar, I. Guerrero and A. Najera for helping with the field work; J. Rivera and P. González for their assistance with GIS to estimate coverages; C. Arguelles for technical support; and F. Heredia and local fishermen for their personal support. E. Glazier and G.E. Donohoe edited the English-language text.

\section{REFERENCES}

Bancroft, G. (1927). Breeding birds at Scammon's Lagoon, Lower California. Condor, 29: 29-57.

Breceda, A., Castellanos, A., Arriaga, L. and OrtegaRubio, A. (1995). Nature conservation at Baja California Sur, Mexico: Protected areas. J. Natural Areas, 15: 267-273.

Carmona, R. and Danemann, G. (1998). Spatiotemporal distribution of birds at the Guerrero Negro saltworks, Baja California Sur, Mexico. Ciencias Marinas, 24(4): 389-408.

Castellanos, A. (1983). Observaciones sobre distribución, abundancia y productividad del águila pescadora en la Laguna Ojo de Liebre-Guerrero Negro, Baja California Sur, México. En: Reunión sobre "La Fauna y su Medio Ambiente", Noroeste de Mexico-Suroeste de Estados Unidos de América. Gen. Tech. Rep. WO-36S. USDA Forest Service, Washington, DC, pp. $98-103$.

Castellanos, A. and Ortega-Rubio, A. (1995). Artificial nesting sites and ospreys at Ojo de Liebre and Guerrero Negro lagoons, Baja California Sur, Mexico. J. Field Ornithol., 66: 117-127.

Castellanos, A., Salinas-Zavala, F. and OrtegaRubio, A. (1994a). Status and reproduction of the peregrine falcon at a coastal lagoon in Baja California Sur, Mexico. J. Raptor Res., 28: 110-112.

Castellanos, A., Salinas-Zavala, F. and OrtegaRubio, A. (1994b). First nesting record of the laughing gull for the west coast of Baja California, Mexico. Western Birds, 25: 203-205. abiertas de ambas lagunas, sino en los salitrales adyacentes y esta actividad también ha incrementado el hábitat accesible para las aves acuáticas en la región (ver Ortega-Rubio et al., 1998; Carmona y Danemann, 1998; Danemann y Carmona, 2000); (iii) la restricción a asentamientos humanos y a cualquier actividad intensiva y de gran escala en las lagunas, basada en los decretos del refugio y la reserva y en la visión de las autoridades de las áreas protegidas como un medio para proteger la naturaleza; y (iv) una zonificación de facto de no desarrollo debido a que la mayor parte de las áreas que rodean a las lagunas pertenecen a la concesión minera de ESSA y están estrictamente bajo su control.

Los pocos sitios de anidamiento originalmente localizados en las marismas fueron abandonados, probablemente debido a la perturbación humana después de que la región se pobló a mediados de los años cuarenta. El saqueo de nidos y el tránsito de lanchas pudieron haber jugado un papel importante en esto. Las aves se pudieron haber cambiado hacia las islas. Sin embargo, las especies localizadas en 1926 en las islas, excepto la gallina de agua y la golondrina marina elegante, todavía anidan ahí, presentando poblaciones estables o un incremento en sus números. Algunas de estas especies incluso han ampliado su zona de anidamiento más allá de las islas.

Los hábitats de anidamiento naturales accesibles son escasos para la mayoría de las aves acuáticas coloniales y algunos substratos están desapareciendo debido a la erosión por el oleaje. La reocupación de las marismas por las garzas como sitios de anidamiento en las lagunas parece poco viable, debido al actual nivel de tránsito de lanchas. Sin embargo, varias especies comenzaron a anidar en los estanques salinos y en el paisaje urbano, los cuales representan hábitats de anidamiento alternativos.

Nosotros creemos que el estatus de reserva de la biósfera del área puede proporcionar el 
Castellanos, A., Jaramillo, F., Salinas, F., OrtegaRubio, A. and Arguelles, C. (1997). Peregrine falcon recovery along the west central coast of the Baja California peninsula, Mexico. J. Raptor Res., 31(1): 1-6.

Cowardin, L., Carter, V., Golet, F. and LaRoe, E.T. (1979). Classification of wetlands and deepwater habitats of the United States. US Dept. Interior, Fish and Wildlife Serv. Res. Pub. 138. Washington, DC, $151 \mathrm{pp}$.

Danemann, G. and Carmona, R. (2000). Breeding birds of the Guerrero Negro saltworks, Baja California Sur, Mexico. Western Birds, 31: 195-199.

DUMAC (1995). Landsat TM digital image of Laguna Ojo de Liebre wetlands and open waters coverages, scale 1:50000. Ducks Unlimmited de Mexico, A.C.-PROESTEROS

Everett, W.T. and Anderson, D.W. (1991). Breeding seabirds of Baja California and the Gulf of California. In: J. Croxall (ed.), Seabirds Status and Conservation: A supplement. Tech. Publ. No. 11, International Council on Bird Preservation, pp. 115-139.

Grinnell, J. (1928). A distributional summation of the ornithology of Lower California. Univ. Calif. Publ. Zool., 32: 1-300.

Henny, C.J. and Anderson, D.W. (1979). Osprey distribution, abundance, and status in western North America. III. Baja California and Gulf of California population. Bull. South. Calif. Acad. Sci., 78: 89-106.

Jehl, J. (1977). History and president (sic) status of osprey in northwestern Baja California. In: J.C. Ogden (ed.), Transactions of the North American Osprey Research Conference, National Park Service Trans. Proc. Ser., No. 2., pp. 241-245.

Kenyon, K. (1947). Breeding population of the osprey in Lower California. Condor, 49: 152-158.

Lewis, L.R. and Ebeling, P.E. (1974). Sea guide. Vol. II. Baja California. SEA Publications, Newport Beach, California, 368 pp.

Massey, W.B. (1977). Occurrence and nesting of the least tern and other endangered species in Baja California, Mexico. Western Birds, 8: 67-70. marco estratégico y los recursos necesarios para la conservación de las aves acuáticas residentes. Recomendamos que lo siguiente sea considerado en cualquier estrategia de conservación para el área: (i) proteger a las aves acuáticas residentes como un elemento esencial de la biodiversidad regional, dentro de un enfoque multiespecífico, integrando su manejo en una sola unidad compuesta por el humedal natural y artificial (la salina); (ii) proporcionar protección inmediata contra depredadores a los sitios de anidamiento en las islas Piedra, Alambre y Cholla, mediante el monitoreo antes y durante la estación reproductora, la remoción física de los depredadores y el cercado de las potenciales rutas de acceso de los mismos; (iii) controlar la erosión por el oleaje y rehabilitar el hábitat perdido en la Isla Concha (existe la maquinaria y la capacidad técnica en la ESSA para tal tarea); (iv) evitar la perturbación humana en los sitios de anidamiento localizados en estructuras hechas por el hombre, en el paisaje urbano y en la salina; (v) proteger contra la erosión por el oleaje a los nuevos sitios de anidamiento dentro de los estanques salinos y evaluar la posibilidad de crear isletas potenciales de anidamiento más grandes; (vi) incrementar el interés del público en estas aves, mediante programas de educación ambiental; (vii) investigar y desarrollar mecanismos de uso no consuntivo de las aves acuáticas residentes para proporcionar beneficios económicos a los pobladores de la reserva; $\mathrm{y}$ (viii) promover enfoques consensuales y participativos entre los poseedores del suelo (pescadores, agencias de turismo, ESSA, ejidatarios, agricultores) y las autoridades de la reserva para lograr objetivos de conservación y desarrollo basados en las aves acuáticas residentes.

\section{AGRADECIMIENTOS}

Agradecemos al CONACYT el apoyo financiero para esta investigación, a la 
Massey, W.B. and Palacios, E. (1994). Avifauna of the wetlands of Baja California, Mexico: Current status. Stud. Avian Biol., 15: 45-57.

Ortega-Rubio, A., Castellanos, A. and Lluch-Cota., D. (1998). Sustainable development in a Mexican biosphere reserve: Salt production in Vizcaíno, Baja California (Mexico). J. Natural Areas, 18: 63-72.

Salinas-Zavala, C.A., Llinas, J. and RodríguezEstrella, R. (1991). Aspectos biológicos del águila pescadora (Pandion haliaetus carolinensis). En: A. Ortega y L. Arriaga (eds.), La Reserva de la Biósfera del Vizcaíno en la Península de Baja California. Centro de Investigaciones Biológicas, La Paz, Baja California Sur, pp. 265-293.

Saunders, G. and Saunders, D. (1981). Winter waterfowl and their wintering grounds in Mexico, 1937-64. US Dept. Interior, Fish and Wildlife Serv. Res. Pub. 138. Washington, DC, $151 \mathrm{pp}$.

Soulé, M. (1991). Conservation: Tactics for a constant crisis. Science, 253: 744-750.
SEMARNAP y ESSA su apoyo institucional, y a S.R. Osorio y E. Palacios sus datos no publicados. Agradecemos a E. Palacios y dos revisores anónimos la revisión crítica del manuscrito; a P. Galina, I. Tovar, I. Guerrero y A. Najera su ayuda en el trabajo de campo; a J. Rivera y P. Zamorano su asistencia con el SIG para estimar las coberturas; a C. Arguelles su apoyo técnico; y a F. Heredia y los pescadores locales su apoyo personal. E. Glazier y G.E. Donohoe editaron el texto en inglés.

Traducido al español por los autores.

Wilbur, S.R. (1987). Birds of Baja California. Univ. California Press, Berkeley, 253 pp.

Wilson, M. and Ryan, D.A. (1996). Conservation of Mexican wetlands: Role of the North American Wetlands Conservation Act. Wildlife Soc. Bull., 25: 57-64. 